CLNS 94/1267

CLEO 94-2

September 23, 2021

\title{
Measurement of the branching fraction for $D^{+} \rightarrow K^{-} \pi^{+} \pi^{+}$
}

R. Balest,${ }^{1}$ K. Cho, ${ }^{1}$ M. Daoudi, ${ }^{1}$ W.T. Ford,${ }^{1}$ D.R. Johnson, ${ }^{1}$ K. Lingel, ${ }^{1}$ M. Lohner, ${ }^{1}$ P. Rankin, ${ }^{1}$ J.G. Smith, ${ }^{1}$ J.P. Alexander,${ }^{2}$ C. Bebek,${ }^{2}$ K. Berkelman,${ }^{2}$ K. Bloom,${ }^{2}$ T.E. Browder, ${ }^{2 *}$ D.G. Cassel,${ }^{2}$ H.A. Cho, ${ }^{2}$ D.M. Coffman, ${ }^{2}$ P.S. Drell, ${ }^{2}$ R. Ehrlich, ${ }^{2}$ P. Gaiderev, ${ }^{2}$ M. Garcia-Sciveres, ${ }^{2}$ B. Geiser, ${ }^{2}$ B. Gittelman, ${ }^{2}$ S.W. Gray,${ }^{2}$ D.L. Hartill, ${ }^{2}$ B.K. Heltsley, ${ }^{2}$ C.D. Jones, ${ }^{2}$ S.L. Jones,${ }^{2}$ J. Kandaswamy, ${ }^{2}$ N. Katayama, ${ }^{2}$ P.C. Kim,${ }^{2}$ D.L. Kreinick, ${ }^{2}$ G.S. Ludwig, ${ }^{2}$ J. Masui, ${ }^{2}$ J. Mevissen,${ }^{2}$ N.B. Mistry, ${ }^{2}$ C.R. Ng, ${ }^{2}$ E. Nordberg, ${ }^{2}$ J.R. Patterson, ${ }^{2}$ D. Peterson, ${ }^{2}$ D. Riley,${ }^{2}$ S. Salman, ${ }^{2}$ M. Sapper,${ }^{2}$ F. Würthwein, ${ }^{2}$ P. Avery, ${ }^{3}$ A. Freyberger,${ }^{3}$ J. Rodriguez, ${ }^{3}$ R. Stephens, ${ }^{3}$ S. Yang, ${ }^{3}$ J. Yelton, ${ }^{3}$ D. Cinabro, ${ }^{4}$ S. Henderson,${ }^{4}$ T. Liu ${ }^{4}$ M. Saulnier, ${ }^{4}$ R. Wilson, ${ }^{4}$ H. Yamamoto,${ }^{4}$ T. Bergfeld, ${ }^{5}$ B.I. Eisenstein, ${ }^{5}$ G. Gollin, ${ }^{5}$ B. Ong,${ }^{5}$ M. Palmer,${ }^{5}$ M. Selen, ${ }^{5}$ J. J. Thaler,${ }^{5}$ A.J. Sadoff ${ }^{6}$ R. Ammar, ${ }^{7}$ S. Ball, ${ }^{7}$ P. Baringer, ${ }^{7}$ A. Bean,${ }^{7}$ D. Besson,${ }^{7}$ D. Coppage, ${ }^{7}$ N. Copty,${ }^{7}$ R. Davis, ${ }^{7}$ N. Hancock, ${ }^{7}$ M. Kelly, ${ }^{7}$ N. Kwak, ${ }^{7}$ H. Lam,${ }^{7}$ Y. Kubota, ${ }^{8}$ M. Lattery, ${ }^{8}$ J.K. Nelson,${ }^{8}$ S. Patton, ${ }^{8}$ D. Perticone,${ }^{8}$ R. Poling, ${ }^{8}$ V. Savinov,${ }^{8}$ S. Schrenk ${ }^{8}$

R. Wang, ${ }^{8}$ M.S. Alam, ${ }^{9}$ I.J. Kim,,${ }^{9}$ B. Nemati, ${ }^{9}$ J.J. O'Neill, ${ }^{9}$ H. Severini,${ }^{9}$ C.R. Sun,${ }^{9}$ M.M. Zoeller, ${ }^{9}$ G. Crawford,${ }^{10}$ C. M. Daubenmier,${ }^{10}$ R. Fulton,${ }^{10}$ D. Fujino, ${ }^{10}$ K.K. Gan,${ }^{10}$ K. Honscheid,${ }^{10}$ H. Kagan,${ }^{10}$ R. Kass,${ }^{10}$ J. Lee,${ }^{10}$ R. Malchow,${ }^{10}$ Y. Skovpen,${ }^{10 \dagger}$ M. Sung,${ }^{10}$ C. White, ${ }^{10}$ F. Butler, ${ }^{11}$ X. Fu,${ }^{11}$ G. Kalbfleisch,,${ }^{11}$ W.R. Ross,${ }^{11}$ P. Skubic,${ }^{11}$ J. Snow, ${ }^{11}$ P.L. Wang, ${ }^{11}$ M. Wood,${ }^{11}$ D.N. Brown,${ }^{12}$ J.Fast,${ }^{12}$ R.L. McIlwain, ${ }^{12}$ T. Miao, ${ }^{12}$ D.H. Miller, ${ }^{12}$ M. Modesitt,${ }^{12}$ D. Payne,${ }^{12}$ E.I. Shibata, ${ }^{12}$ I.P.J. Shipsey ${ }^{12}$ P.N. Wang ${ }^{12}$ M. Battle ${ }^{13}$ J. Ernst, ${ }^{13}$ Y. Kwon, ${ }^{13}$ S. Roberts, ${ }^{13}$ E.H. Thorndike, ${ }^{13}$ C.H. Wang, ${ }^{13}$ J. Dominick, ${ }^{14}$ M. Lambrecht, ${ }^{14}$ S. Sanghera, ${ }^{14}$ V. Shelkov, ${ }^{14}$ T. Skwarnicki, ${ }^{14}$ R. Stroynowski, ${ }^{14}$ I. Volobouev,${ }^{14}$ G. Wei,${ }^{14}$ P. Zadorozhny, ${ }^{14}$ M. Artuso ${ }^{15}$ M. Goldberg, ${ }^{15}$

D. He,${ }^{15}$ N. Horwitz,${ }^{15}$ R. Kennett,${ }^{15}$ R. Mountain,${ }^{15}$ G.C. Moneti ${ }^{15}$ F. Muheim,${ }^{15}$ Y. Mukhin,${ }^{15}$ S. Playfer,${ }^{15}$ Y. Rozen,${ }^{15}$ S. Stone,${ }^{15}$ M. Thulasidas, ${ }^{15}$ G. Vasseur,${ }^{15}$ G. Zhu, ${ }^{15}$ J. Bartelt, ${ }^{16}$ S.E. Csorna,${ }^{16}$ Z. Egyed,${ }^{16}$ V. Jain, ${ }^{16}$ K. Kinoshita,${ }^{17}$ K.W. Edwards,,${ }^{18}$ M. Ogg ${ }^{18}$ D.I. Britton, ${ }^{19}$ E.R.F. Hyatt, ${ }^{19}$ D.B. MacFarlane, ${ }^{19}$ P.M. Patel ${ }^{19}$ D.S. Akerib,${ }^{20}$ B. Barish, ${ }^{20}$ M. Chadha,${ }^{20}$ S. Chan, ${ }^{20}$ D.F. Cowen,${ }^{20}$ G. Eigen, ${ }^{20}$ J.S. Miller ${ }^{20}$ C. O'Grady ${ }^{20}$ J. Urheim, ${ }^{20}$ A.J. Weinstein, ${ }^{20}$ D. Acosta, ${ }^{21}$ M. Athanas, ${ }^{21}$ G. Masek,${ }^{21}$ H.P. Paar ${ }^{21}$ J. Gronberg, ${ }^{22}$ R. Kutschke, ${ }^{22}$ S. Menary, ${ }^{22}$ R.J. Morrison, ${ }^{22}$ S. Nakanishi, ${ }^{22}$ H.N. Nelson, ${ }^{22}$ T.K. Nelson, ${ }^{22}$ C. Qiao, ${ }^{22}$ J.D. Richman, ${ }^{22}$ A. Ryd ${ }^{22}$ H. Tajima,${ }^{22}$ D. Sperka, ${ }^{22}$ M.S. Witherell, ${ }^{22}$ and M. Procario ${ }^{23}$

\author{
(CLEO Collaboration)
}


${ }^{1}$ University of Colorado, Boulder, Colorado 80309-0390

${ }^{2}$ Cornell University, Ithaca, New York 14853

${ }^{3}$ University of Florida, Gainesville, Florida 32611

${ }^{4}$ Harvard University, Cambridge, Massachusetts 02138

${ }^{5}$ University of Illinois, Champaign-Urbana, Illinois, 61801

${ }^{6}$ Ithaca College, Ithaca, New York 14850

${ }^{7}$ University of Kansas, Lawrence, Kansas 66045

${ }^{8}$ University of Minnesota, Minneapolis, Minnesota 55455

${ }^{9}$ State University of New York at Albany, Albany, New York 12222

${ }^{10}$ Ohio State University, Columbus, Ohio, 43210

${ }^{11}$ University of Oklahoma, Norman, Oklahoma 73019

${ }^{12}$ Purdue University, West Lafayette, Indiana 47907

${ }^{13}$ University of Rochester, Rochester, New York 14627

${ }^{14}$ Southern Methodist University, Dallas, Texas 75275

${ }^{15}$ Syracuse University, Syracuse, New York 13244

${ }^{16}$ Vanderbilt University, Nashville, Tennessee 37235

${ }^{17}$ Virginia Polytechnic Institute and State University, Blacksburg, Virginia, 24061

${ }^{18}$ Carleton University, Ottawa, Ontario K1S 5B6 and the Institute of Particle Physics, Canada

${ }^{19}$ McGill University, Montréal, Québec H3A 2 T8 and the Institute of Particle Physics, Canada

${ }^{20}$ California Institute of Technology, Pasadena, California 91125

${ }^{21}$ University of California, San Diego, La Jolla, California 92093

${ }^{22}$ University of California, Santa Barbara, California 93106

${ }^{23}$ Carnegie-Mellon University, Pittsburgh, Pennsylvania 15213

(September 23, 2021)

\begin{abstract}
Using the CLEO-II detector at CESR we have measured the ratio of branching fractions, $\mathcal{B}\left(D^{+} \rightarrow K^{-} \pi^{+} \pi^{+}\right) / \mathcal{B}\left(D^{0} \rightarrow K^{-} \pi^{+}\right)=2.35 \pm 0.16 \pm 0.16$. Our recent measurement of $\mathcal{B}\left(D^{0} \rightarrow K^{-} \pi^{+}\right)$then gives $\mathcal{B}\left(D^{+} \rightarrow K^{-} \pi^{+} \pi^{+}\right)=$ $(9.3 \pm 0.6 \pm 0.8) \%$.
\end{abstract}

PAC numbers:13.20.Fc, 13.25.Ft, 14.40.Lb

Typeset using REVTEX

\footnotetext{
*Permanent address: University of Hawaii at Manoa

${ }^{\dagger}$ Permanent address: INP, Novosibirsk, Russia
} 
The decay $D^{+} \rightarrow K^{-} \pi^{+} \pi^{+}$is the most commonly used mode for normalizing $D^{+}$ yields, since it has a relatively large branching fraction and is one of the simplest to reconstruct. Many current charm and bottom meson decay results are limited by the precision of $\mathcal{B}\left(D^{+} \rightarrow K^{-} \pi^{+} \pi^{+}\right)$. Previous measurements of this decay mode were performed by the Mark III [1] and ACCMOR [2] collaborations. Mark III used the relative number of singly detected $D^{ \pm}$mesons to the number of reconstructed $D^{+} D^{-}$events to determine the branching fraction. The ACCMOR collaboration measured the ratio of $D^{+} \rightarrow K^{-} \pi^{+} \pi^{+}$relative to the total number of 3-prong decays, and used topological branching ratios determined by other experiments to obtain a branching fraction. However, ACCMOR could not easily distinguish $D^{+}, D_{s}^{+}$and $\Lambda_{c}^{+}$decay vertices, and had to rely on estimates of the relative production ratios of these particles. In this analysis, we use the exclusive yields, $N_{K \pi}$ and $N_{K \pi \pi}$, of the $\left(D^{*+} \rightarrow D^{0} \pi^{+}, D^{0} \rightarrow K^{-} \pi^{+}\right)$and the $\left(D^{*+} \rightarrow D^{+} \pi^{0}, D^{+} \rightarrow K^{-} \pi^{+} \pi^{+}\right)$decay sequences, respectively, to measure the ratio, $\mathcal{B}\left(D^{+} \rightarrow K^{-} \pi^{+} \pi^{+}\right) / \mathcal{B}\left(D^{0} \rightarrow K^{-} \pi^{+}\right)$, and apply our measurement of the branching fraction for $\mathcal{B}\left(D^{0} \rightarrow K^{-} \pi^{+}\right)$[3] to obtain $\mathcal{B}\left(D^{+} \rightarrow K^{-} \pi^{+} \pi^{+}\right)$.

The data used in this analysis consist of $1.79 \mathrm{fb}^{-1}$ of $e^{+} e^{-}$collisions recorded with the CLEO-II detector operating at the Cornell Electron Storage Ring (CESR). The CLEOII detector has been described in detail elsewhere [4]. Data were recorded at the $\Upsilon(4 S)$ resonance and in the continuum both below and above (the $e^{+} e^{-}$center of mass energies ranged from 10.52 to $10.70 \mathrm{GeV}$ ).

We obtain clean samples of $D^{*}$ mesons by requiring the $\pi^{0}$ and the $\pi^{+}$emitted in their decays to fulfill strict selection criteria. To reconstruct $\pi^{0}$ 's, we start with neutral showers which satisfy isolation cuts and cannot be matched to any charged track in the event. These photons candidates must have $\left|\cos \theta_{\gamma}\right| \leq 0.71,\left(\theta_{\gamma}\right.$ is the polar angle measured relative to the beam axis) to ensure that they lie in that portion of the electromagentic calorimeter which has the best efficiency and resolution, and the least systematic uncertainty. In addition, photon energies have to be greater than $30 \mathrm{MeV}$. We then kinematically constrain $\gamma \gamma$ combinations with masses between 125 and $145 \mathrm{MeV} / \mathrm{c}^{2}$ to the known $\pi^{0}$ mass to improve the momentum resolution. To reduce $\gamma \gamma$ combinatoric background, $\pi^{0}$ candidates are required to have momenta greater than $200 \mathrm{MeV} / \mathrm{c}$. In addition, the kinematically constrained $\pi^{0}$ candidates must have $\left|\cos \theta_{\pi^{0}}\right| \leq 0.70$. Charged pions are selected if they have momentum greater than $200 \mathrm{MeV} / \mathrm{c}$, and $\left|\cos \theta_{\pi^{ \pm}}\right| \leq 0.70$. The polar angle cuts on $\pi^{+}$'s and $\pi^{0}$ 's ensure that $D^{*+}$ mesons reconstructed with either charged or neutral pions have the same geometric acceptance.

The ratio $N_{K \pi \pi} / N_{K \pi}$ of the measured yields can be expressed in terms of branching ratios and efficiencies as,

$$
\frac{N_{K \pi \pi}}{N_{K \pi}}=\frac{N_{D^{*+}} \mathcal{B}\left(D^{*+} \rightarrow D^{+} \pi^{0}\right) \mathcal{B}_{K \pi \pi} \epsilon_{K \pi \pi}}{N_{D^{*+}} \mathcal{B}\left(D^{*+} \rightarrow D^{0} \pi^{+}\right) \mathcal{B}_{K \pi} \epsilon_{K \pi}}
$$

where $\mathcal{B}_{K \pi \pi}$ and $\mathcal{B}_{K \pi}$ are the relevant $D^{+}$and $D^{0}$ branching fractions, respectively. The total number of $D^{*+}$ 's produced in the data sample is $N_{D^{*+}}$ (which cancels in the ratio); $\epsilon_{K \pi \pi}$ and $\epsilon_{K \pi}$ are the efficiencies for reconstructing $D^{+} \rightarrow K^{-} \pi^{+} \pi^{+}$and $D^{0} \rightarrow K^{-} \pi^{+}$, respectively, with their respective $D^{*+}$ tags. Using isospin invariance, the CLEO-II measurements of the $D^{*+}-D^{+}$and $D^{*+}-D^{0}$ mass differences [5], and the fact that these decays are p-wave, we estimate the ratio [6] 


$$
\frac{\mathcal{B}\left(D^{*+} \rightarrow \pi^{+} D^{0}\right)}{\mathcal{B}\left(D^{*+} \rightarrow \pi^{0} D^{+}\right)}=2.21 \pm 0.07
$$

The efficiencies in Eq. (1) include the efficiency of reconstructing the $D$ decay, as well as the efficiency for the slow pion emitted in the $D^{*}$ decay. The $D$ reconstruction efficiency is reliably simulated by the Monte Carlo because the main cuts on the $D$ daughters are geometric. It is harder to simulate the efficiencies to detect the slow neutral and charged pions, since the efficiency for detecting charged tracks varies rapidly at low momentum [7], and the $\pi^{0}$ efficiency is known only to $\pm 5 \%$. We have checked the slow charged and neutral pion efficiencies from the data in several ways. The ratio of branching fractions for $\eta \rightarrow \pi^{0} \pi^{0} \pi^{0}$ and $\eta \rightarrow \gamma \gamma$ as measured in our data sample has been compared with the world average [8] to obtain an estimate of the accuracy of the photon finding efficiency. We find that this efficiency is simulated to an accuracy of $\pm 2.5 \%$. We have studied the charged particle tracking by comparing the yield of fully reconstructed $D^{0} \rightarrow K^{-} \pi^{+} \pi^{0}$ with partially reconstructed $D^{0} \rightarrow K^{-} \pi^{0}\left(\pi^{+}\right)$, where the $\pi^{+}$is not detected. This check shows that the charged pion efficiency is simulated to a precision which is better than $\pm 2 \%$.

In addition, we have directly checked the ratio of slow neutral and charged pion efficiencies, $\epsilon_{\pi^{0}} / \epsilon_{\pi^{+}}$, from the data. Using yields for $D^{*+(0)} \rightarrow D^{0} \pi^{+(0)}$, where $D^{0} \rightarrow K^{-} \pi^{+}$, we have measured the ratio of inclusive $D^{*+}$ and $D^{* 0}$ production cross-section in the continuum to be $1.06 \pm 0.09$. This result is consistent with unity, which is expected since the $D^{*}$ 's are not being produced near threshold and the $D^{*+}-D^{* 0}$ mass difference is negligible compared to the center of mass energy. We have also studied this ratio by using the decays $\eta \rightarrow \pi^{+} \pi^{-} \pi^{0}$ and $K_{s}^{0} \rightarrow \pi^{+} \pi^{-}, \pi^{0} \pi^{0}$; here we find that the Monte Carlo estimate for $\epsilon_{\pi^{0}} / \epsilon_{\pi^{+}}$is $0.96 \pm 0.05$ times the value extracted from data. In summary, all our checks of the estimate for $\epsilon_{\pi^{0}} / \epsilon_{\pi^{+}}$ show that there is good agreement between Monte Carlo and data. We assign a systematic error of $\pm 5.4 \%$ due to this efficiency ratio in the final results.

We now determine the exclusive yields and reconstruction efficiencies used in Eq. (11). The $D^{+}$is reconstructed by requiring the $K^{-}$and $\pi^{+}$tracks to have $|\cos \theta| \leq 0.81$, and to have momenta greater than $200 \mathrm{MeV} / \mathrm{c}$. In Fig. 1 we present the invariant mass distribution of $K^{-} \pi^{+} \pi^{+}$combinations, for which the mass difference, $\Delta_{M^{0}}=M_{K^{-} \pi^{+} \pi^{+}\left(\pi^{0}\right)}-M_{K^{-} \pi^{+} \pi^{+}}$, is within $\pm 5 \mathrm{MeV} / c^{2}$ of the value expected for $M_{D^{*+}}-M_{D^{+}}$. Using two Gaussians for the signal and a first order polynomial for the background, we obtain $1618 \pm 91$ events. To account for true $D^{+}$- random $\pi^{0}$ combinations, we also fit the $\Delta_{M^{0}}$ sidebands. We observe $116 \pm 40$ events in the scaled sideband [9]. After subtraction, the net yield is $1502 \pm 99$ events. The efficiency $\epsilon_{K \pi \pi}$ is estimated from Monte Carlo to be $(15.4 \pm 0.2) \%$.

The $D^{0}$ is tagged using the $D^{*+} \rightarrow D^{0} \pi^{+}$decay, and is reconstructed by requiring the $K^{-}$and $\pi^{+}$tracks to have $|\cos \theta| \leq 0.81$, and to have momenta greater than $200 \mathrm{MeV} / \mathrm{c}$. In Fig. 2 we present the invariant mass distribution of $K^{-} \pi^{+}$combinations, where the mass difference, $\Delta_{M^{+}}=M_{K^{-} \pi^{+}\left(\pi^{+}\right)}-M_{K^{-} \pi^{+}}$, is within $\pm 5 \mathrm{MeV} / c^{2}$ of the value expected for $M_{D^{*+}}-M_{D^{0}}$. Using two Gaussians for the signal and a first order polynomial for the background, we obtain $5555 \pm 102$ events. A fit to the $\Delta_{M^{+}}$sidebands yields $103 \pm 21$ events [9]. The net yield is $5452 \pm 104$ events. The efficiency $\epsilon_{K \pi}$ is estimated to be $(59.5 \pm 0.6) \%$.

Substituting the exclusive yields, the efficiencies for reconstructing these final states, and the known ratio of $D^{*+} \rightarrow D \pi$ branching fractions in Eq. (四), we obtain 


$$
\frac{\mathcal{B}\left(D^{+} \rightarrow K^{-} \pi^{+} \pi^{+}\right)}{\mathcal{B}\left(D^{0} \rightarrow K^{-} \pi^{+}\right)}=2.35 \pm 0.16 \pm 0.16
$$

where the first error is statistical and the second is an estimate of the systematic uncertainty. The systematic error includes the uncertainty on the ratio of $D^{*+} \rightarrow D \pi$ branching fractions $( \pm 3.2 \%)$, the error due to $\epsilon_{\pi^{0}} / \epsilon_{\pi^{+}}( \pm 5.4 \%)$, the error due to Monte Carlo statistics $( \pm 1.6 \%)$, and the uncertainty from the effects of resonant substructure on the $K \pi \pi$ final state $( \pm 1.3 \%)$. Using our measurement [3], $\mathcal{B}\left(D^{0} \rightarrow K^{-} \pi^{+}\right)=(3.95 \pm 0.08 \pm 0.17) \%$, we obtain

$$
\mathcal{B}\left(D^{+} \rightarrow K^{-} \pi^{+} \pi^{+}\right)=(9.3 \pm 0.6 \pm 0.8) \%
$$

This result accounts for the effects of decay radiation in the final state, because we have used the radiatively corrected value for $\mathcal{B}\left(D^{0} \rightarrow K^{-} \pi^{+}\right)$[10]. The determination of statistical and systematic errors is described above. The systematic error also includes the error in our measurement of $\mathcal{B}\left(D^{0} \rightarrow K^{-} \pi^{+}\right)$.

In conclusion, using yields of $\left(D^{+} \rightarrow K^{-} \pi^{+} \pi^{+}\right)$and $\left(D^{0} \rightarrow K^{-} \pi^{+}\right)$, which have been tagged via $D^{*+} \rightarrow D \pi$ decays, and our measurement of $\mathcal{B}\left(D^{0} \rightarrow K^{-} \pi^{+}\right.$) [3], we obtain $\mathcal{B}\left(D^{+} \rightarrow K^{-} \pi^{+} \pi^{+}\right)=(9.3 \pm 0.6 \pm 0.8) \%$. This result agrees well with the Mark III measurement, $(9.1 \pm 1.3 \pm 0.4) \%$ [1], but is larger than the ACCMOR result, $(6.4 \pm 1.5) \%$ [2].

We gratefully acknowledge the effort of the CESR staff in providing us with excellent luminosity and running conditions. This work was supported by the National Science Foundation, the U.S. Dept. of Energy, the SSC Fellowship of TNRLC, the Heisenberg Foundation and the A.P. Sloan Foundation. 


\section{REFERENCES}

[1] Mark III Collaboration, J. Adler et al., Phys. Rev. Lett. 60, 89 (1988)

[2] ACCMOR Collaboration, S. Barlag et al., Z. Phys. C55, 383 (1992); S. Barlag et al., Z. Phys. C48, 29 (1990).

[3] CLEO Collaboration, D. Akerib et al., Phys. Rev. Lett. 71, 3070 (1993).

[4] CLEO Collaboration, Y. Kubota et al., Nucl. Intr. and Meth. A320, 66 (1992).

[5] CLEO Collaboration, D. Bortoletto et al., Phys. Rev. Lett. 69, 2046 (1992).

[6] CLEO Collaboration, F. Butler et al., Phys. Rev. Lett. 69, 2041 (1992).

[7] The average momentum for slow pions emitted in $D^{*}$ decays which pass the selection criteria is approximately $260 \mathrm{MeV} / \mathrm{c}$, whereas the average momenta of the $D$ decay daughters is approximately $1.3 \mathrm{GeV} / \mathrm{c}$ for $D^{+}$and $1.7 \mathrm{GeV} / \mathrm{c}$ for $D^{0}$. The efficiency for reconstructing kaons and pions from $D$ decays is known to an accuracy of $\pm 2 \%$ [3].

[8] Particle Data Group, K. Hikasa et al., Phys. Rev. 45, 1 (1992).

[9] The scale factor is such that the number of events in the sideband region is the same as the number of events in the background under the mass difference signal. We investigated different mass difference sidebands to estimate the systematic error associated with this technique. The yields quoted in the text are obtained from the sideband where the $D^{*+}-D$ mass difference is in the range $146.6-155.6 \mathrm{MeV} / c^{2}$ for $D^{*+}-D^{+}$, and 151.4 - $160.4 \mathrm{MeV} / c^{2}$ for $D^{*+}-D^{0}$.

[10] The effect of final state decay radiation on $D^{0} \rightarrow K^{-} \pi^{+}$and $D^{+} \rightarrow K^{-} \pi^{+} \pi^{+}$is to reduce their reconstruction efficiencies by approximately $1 \%$. Therefore, the ratio of the two efficiencies, $\epsilon_{K \pi}$ and $\epsilon_{K \pi \pi}$ is very insensitive to the effects of decay radiation. We used the program PHOTOS to estimate these effects - E. Barberio, B. van Eijk and Z. Was, Comput. Phys. Commun. 66115 (1991). 


\section{FIGURES}

FIG. 1. Mass distribution for $D^{+} \rightarrow K^{-} \pi^{+} \pi^{+}$candidates tagged via $D^{*+} \rightarrow D^{+} \pi^{0}$ decays; the histogram represent events in the mass difference signal region, triangles with error bars represent events in the (scaled) mass difference sideband region. The solid line is the fit to the data.

FIG. 2. Mass distribution for $D^{0} \rightarrow K^{-} \pi^{+}$tagged via $D^{*+} \rightarrow D^{0} \pi^{+}$decays; the histogram represent events in the mass difference signal region, triangles represent events in the (scaled) mass difference sideband region. The solid line is the fit to the data. 
This figure "fig1-1.png" is available in "png" format from: http://arxiv.org/ps/hep-ph/9403382v1 
This figure "fig1-2.png" is available in "png" format from: http://arxiv.org/ps/hep-ph/9403382v1 